\title{
La calidad del agua para consumo humano y su asociación con la morbimortalidad en Colombia, 2008-2012
}

\author{
Blanca Lisseth Guzmán', Gerardo Nava1, Paula Dias Bevilacqua² \\ ${ }^{1}$ Grupo de Calidad del Agua, Subdirección Laboratorio Nacional de Referencia, Dirección de Redes en Salud \\ Pública, Instituto Nacional de Salud, Bogotá, D.C., Colombia \\ 2 Departamento de Veterinária, Universidade Federal de Viçosa, Viçosa, Minas Gerais, Brasil
}

\begin{abstract}
Introducción. La calidad del agua para consumo humano se ha asociado con la aparición de diversas enfermedades. El estudio de la relación entre estos eventos permitiría reflejar el impacto de la calidad del agua de consumo en la salud de la población, con el fin de orientar acciones de prevención y promoción en salud ambiental.
\end{abstract}

Objetivo. Analizar la calidad del agua para consumo humano y su asociación con la morbimortalidad en Colombia, 2008-2012.

Materiales y métodos. Se analizó la base de datos de la vigilancia de la calidad del agua mediante métodos de estadística descriptiva en lo referente a las principales características indicadoras de calidad (coliformes totales, Escherichia coli, turbiedad, color, $\mathrm{pH}$, cloro residual libre e índice del riesgo de la calidad del agua). Los resultados se correlacionaron con la mortalidad infantil y la morbilidad por enfermedad diarreica aguda, enfermedades transmitidas por alimentos y hepatitis $\mathrm{A}$. Se elaboró un mapa de riesgo para identificar los municipios con alto riesgo de contaminación del agua y mortalidad infantil.

Resultados. Se encontró un alto porcentaje de municipios en los que el valor de potabilidad del agua no se ajustaba a lo establecido por la norma vigente; se identificaron los problemas relacionados con la presencia de E. coli, de coliformes totales y la ausencia de cloro residual libre, los cuales fueron más agudos en la zona rural. La calidad del agua tuvo una mayor correlación con la mortalidad infantil, constatándose así su importancia para la salud de la población infantil.

Conclusión. La calidad del agua demostró tener un impacto importante en la mortalidad infantil, por lo que se requiere la adopción de políticas que fortalezcan los sistemas de suministro de agua en el país. Es esencial fortalecer los programas de vigilancia en salud ambiental, para orientar las acciones de mejoramiento de la calidad del agua e influir positivamente en la salud.

Palabras clave: calidad del agua, indicadores ambientales, salud ambiental, vigilancia sanitaria ambiental, sistemas de información.

doi: http://dx.doi.org/10.7705/biomedica.v35i0.2511

Quality of water for human consumption and its association with morbimortality in Colombia, 2008-2012

Introduction: The quality of water for human consumption has been correlated with the occurrence of different diseases. Studying the relationship between these parameters would allow determining the impact of water quality on human health, and to direct preventative measures and promote environmental health.

Objective: To analyze the quality of water intended for human consumption and its association with morbimortality in Colombia, 2008-2012.

Materials and methods: The database for surveillance of water quality was analyzed by means of descriptive statistics of the principal indicators (total coliforms, Escherichia coli, turbidity, color, $\mathrm{pH}$, free residual chlorine and water quality risk index). The results were correlated with infant mortality and morbidity due to acute diarrheal diseases, foodborne diseases and hepatitis A. A risk map was prepared to identify those municipalities with the highest risk of water contamination and infant mortality.

Results: A high percentage of municipalities did not conform to existing standards for water potability values. Problems were identified that were related to presence of $E$. coli and total coliforms, as well as absence of free residual chlorine, a situation that was exacerbated in rural areas. Water quality showed a high correlation with infant mortality, highlighting its importance for children's health.

\section{Contribución de los autores:}

Todos los autores participaron en la planeación y la ejecución del estudio, así como en la recolección, organización, análisis e interpretación de los datos, y en la elaboración, redacción y revisión crítica del artículo. 
Conclusion: Water quality was found to have an important impact on infant mortality. Improving water quality in Colombia will require policies that strengthen water supply systems in this country. Strengthening of environmental health surveillance programs is essential to guide actions aimed at improving water quality and exert a positive impact on health.

Key words: Water quality, environmental indicators, environmental health, environmental health surveillance, information systems.

doi: http://dx.doi.org/10.7705/biomedica.v35i0.2511

El agua es una necesidad vital que influye de forma directa en la salud. La calidad del agua de consumo humano se ha asociado con diversas enfermedades. Un gran número de enfermedades infecciosas y parasitarias en el mundo, se debe a la falta de acceso adecuado a fuentes de agua y a condiciones de saneamiento, y la Organización Mundial de la Salud (OMS) estima que 2,9 millones de personas mueren cada año por estas causas (1). Los menores de edad son el grupo de edad más afectado, ya que $90 \%$ de las muertes ocurre en niños menores de cinco años, casi siempre residentes en países en desarrollo (1).

En varios estudios se ha demostrado la asociación entre las condiciones de saneamiento, incluido el acceso a agua potable, con enfermedades diarreicas, especialmente en menores de cinco años. Dicha asociación se ha estudiado bastante y diversos autores la han demostrado a partir de estudios epidemiológicos (2-4). Igualmente, los estudios han demostrado la importancia de las estrategias de saneamiento en la reducción de las enfermedades diarreicas, evidenciado así el impacto positivo de dichas intervenciones en la salud de la población (5-9). Se estima que el $94 \%$ de los casos de diarrea podría evitarse mediante el aumento de la disponibilidad del agua potable y el acceso al saneamiento básico, así como la promoción de acciones de educación en salud (10).

En consecuencia, es fundamental garantizar el acceso a agua potable para mejorar las condiciones de salud de las poblaciones y evitar la trasmisión de enfermedades. El acceso al agua potable hace parte de los Objetivos de Desarrollo del Milenio establecidos por las Naciones Unidas, para lo

\section{Correspondencia:}

Blanca Lisseth Guzmán, Grupo de Calidad del Agua, Subdirección Laboratorio Nacional de Referencia, Dirección de Redes en Salud Pública, Instituto Nacional de Salud, Avenida Calle $26 \mathrm{~N}^{\circ}$ 51-20, Bogotá, D.C., Colombia

Teléfonos: (1) 220 7700, extensión 1295; 3164928712

blancalissethguz@hotmail.com, bguzma@ins.gov.co

Recibido: 14/08/14; aceptado: 26/05/15 cual se plantea la meta de reducir a la mitad el porcentaje de personas sin acceso a agua potable y saneamiento para el año 2015. Los avances en la consecución de dicha meta se reflejan en las cifras: en el 2010 más de dos millones de personas habían obtenido acceso a fuentes de agua mejoradas; sin embargo, todavía hay 780.000 millones de personas sin acceso al agua potable, y persisten grandes diferencias entre regiones (11). En América Latina y el Caribe, cerca de 38 millones de personas no tienen acceso a fuentes de agua potable y las enfermedades de origen hídrico aparecen entre las tres principales causas de muerte, con el consecuente impacto en la salud pública de la región (12). Se han identificado, igualmente, desigualdades significativas en el acceso a los servicios, lo que conlleva el aumento de la exposición a los riesgos ambientales en diferentes regiones y grupos de población (10).

En Colombia, el acceso al agua potable alcanzó una cobertura del $92 \%$ en el 2012, con una importante diferencia entre el $99 \%$ de cobertura en el área urbana y el $72 \%$ en la rural, donde aún hay un elevado porcentaje de la población sin acceso al agua potable (11). Para mejorar esta situación, en el país se han promovido diversas acciones, entre ellas la implementación del Sistema de Vigilancia de la Calidad del Agua para Consumo Humano, estrategia que tiene un amplio reconocimiento mundial $(13,14)$, y cuyo objetivo, según lo establecido en el Decreto 1575 del 2007, es comprobar y evaluar el riesgo en salud pública del agua mediante la vigilancia rutinaria y continua de sus características microbiológicas y fisicoquímicas, con el fin de garantizar a la población agua de calidad acorde con los valores de potabilidad establecidos en la Resolución 2115 del 2007 (15,16). Las actividades de vigilancia buscan aportar el conocimiento pertinente sobre las principales características del agua de consumo, mediante el análisis de la tendencia y la dinámica de las condiciones de la calidad del agua distribuida en los diferentes territorios de Colombia, y así, contribuir al establecimiento de estrategias que permitan mejorarla. 
Las políticas para el sector de salud ambiental en Colombia han avanzado notablemente a partir de la publicación del documento 3550 del 2008 emitido por el CONPES y del Plan Nacional de Salud Pública, 2012-2021. En lo referente al agua, en el plan de gestión integral del recurso hídrico se han establecido diferentes estrategias para mejorar su calidad y reducir las desigualdades en el acceso. Sin embargo, existe poca información sobre el impacto de la calidad del agua para consumo humano en la salud; de ahí la necesidad de llevar a cabo estudios que exploren esta relación en el país, para ofrecer información que oriente las estrategias de mejoramiento de la calidad del agua y respalde las decisiones intersectoriales, locales, regionales y nacionales sobre este recurso.

En este estudio se planteó el objetivo de analizar los resultados de la vigilancia de la calidad del agua a cargo de las autoridades de salud en los territorios de Colombia, así como la asociación entre la calidad del agua, la mortalidad infantil y la morbilidad por la enfermedad diarreica aguda, las enfermedades transmitidas por alimentos y la hepatitis $A$.

\section{Materiales y métodos}

\section{Bases de datos}

La base de datos de la calidad del agua se obtuvo del Sistema de Información de la Vigilancia de la Calidad del Agua para Consumo Humano (SIVICAP), en el cual se reportan los resultados de la vigilancia de la calidad del agua para consumo humano que adelantan las autoridades de salud en los diferentes territorios de Colombia. Esta vigilancia incluye la recolección de muestras, el análisis e interpretación de resultados, el suministro de información y la utilización de esta información en pro de la salud pública (15). Para la recolección de muestras se desarrolla el plan de vigilancia de la calidad del agua establecido en la Resolución 2115 del 2007, y en el cual se establecen la frecuencia y el número de muestras según las características y la población abastecida (16). En el análisis se consideraron las características de color, $\mathrm{pH}$, turbiedad, cloro residual libre, coliformes totales y Escherichia coli, por ser los indicadores más representativos de la calidad microbiológica y fisicoquímica del agua $(13,14)$, y requisito obligatorio para todos los municipios del país. Asimismo, se consideró el índice del riesgo de la calidad del agua, instrumento establecido por la normativa para evaluar la calidad del agua de consumo en el país.
El sistema de vigilancia de la calidad del agua para consumo humano cuenta con el Programa Interlaboratorio de Control de Calidad de Aguas Potables (PICCAP), cuyo objetivo es garantizar la confiabilidad de los resultados del análisis de la calidad del agua. El programa evalúa continuamente la calidad analítica de los diferentes laboratorios encargados de los análisis físicos, químicos y microbiológicos del agua para consumo humano, con base en los lineamientos de las normas NTC-ISO/IEC 17025:2005 y NTC-ISO/ IEC 17043:2010 (17).

Para la definición de los eventos de morbimortalidad, se exploraron las bases de datos del Sivigila y del Departamento Administrativo Nacional de Estadísticas (DANE), y se tuvieron en cuenta las revisiones bibliográficas sobre los eventos relacionados con la calidad del agua $(18,19)$. Los eventos seleccionados fueron la enfermedad diarreica aguda, las enfermedades transmitidas por alimentos y la hepatitis A notificadas en el Sivigila, y la mortalidad infantil reportada en las estadísticas vitales del DANE, debido a la solidez de sus datos y su representatividad territorial (municipios). Se recolectó la información sobre todos estos eventos correspondiente al período comprendido entre 2008 y 2012, a excepción de los datos de mortalidad infantil, que solo estaban disponibles hasta el 2011.

\section{Análisis de datos}

Calidad del agua. Se analizaron los resultados de 211.407 muestras de agua reportadas al SIVICAP entre 2008 y 2012, provenientes de 31 departamentos, y de 1.067 (96,3\%) municipios del total de 1.102 existentes en el país según el DANE; no se incluyó al departamento del Chocó, ya que no reportó información al SIVICAP en este periodo. Inicialmente, se identificaron los datos discrepantes o errores de la base de datos, es decir, aquellos valores considerablemente más altos o más bajos que el conjunto de datos; su identificación se hizo mediante técnicas de estadística descriptiva, considerando la dispersión y la distribución normal de los datos, y calculando el rango entre cuartiles.

En el estudio se abordó el cumplimiento de la norma en lo concerniente a los valores de potabilidad mediante el cálculo del porcentaje de muestras que presentaban resultados entre los valores máximos permitidos (Resolución 2115 de 2007: color, 15 unidades de platino cobalto (UPC); $\mathrm{pH}$ de 6,5-9,0; turbiedad, 5 unidades nefelométricas de turbiedad (UNT); cloro residual líquido, 0,3 a 2,0 
$\mathrm{mg} / \mathrm{L}$; coliformes totales, 0 UFC/100 $\mathrm{cm}^{3}$; E. coli, 0 UFC/100 $\mathrm{cm}^{3}$ ) (16). Posteriormente, los resultados de las características fisicoquímicas se analizaron mediante técnicas de estadística descriptiva, calculando las medidas de posición (valor máximo, valor mínimo, mediana, primer cuartil, tercer cuartil y promedio) y de dispersión (desviación estándar), las cuales se representaron en diagramas de caja para analizar la distribución de los datos.

En cuanto a la presencia de microorganismos, los cálculos se hicieron con el programa SPSS $18^{\circledR}$ (PASW Statistics). Las características microbiológicas no se manejaron de manera descriptiva debido a la gran variabilidad de los resultados y a que el valor máximo permitido es de 0 UFC/100 $\mathrm{cm}^{3}$ para todos.

En cuanto al análisis del índice del riesgo de la calidad del agua, se calculó la media para el total de muestras por año y por municipio, para así obtener el índice general y el municipal. Para la evaluación de la calidad del agua, los valores de este índice por muestras se clasificaron según los niveles establecidos en la Resolución 2115 de 2007: inviable sanitariamente $(80,1$ a $100 \%)$, riesgo alto (35,1 a $80 \%)$, riesgo medio (14,1 a $35 \%)$, riesgo bajo $(5,1$ a $14 \%$ y sin riesgo (0 a $5 \%$ ) (16). Los valores se analizaron descriptivamente mediante series históricas, y se consideraron la distribución geográfica municipal y la zona de recolección (rural o urbana). Posteriormente, los resultados se relacionaron con la posición geográfica en mapas desarrollados mediante el programa ArcView GIS TM, versión 9.3, utilizando como referencia el mapa de la división político-administrativa de Colombia.

Morbimortalidad relacionada con la calidad del agua. Para las enfermedades transmitidas por alimentos, la enfermedad diarreica aguda y la hepatitis $A$, se calcularon las tasas de morbilidad por municipio y se utilizó como numerador el número de casos para todos los grupos de edad. En el caso de las enfermedades transmitidas por alimentos y la enfermedad diarreica aguda, también se analizaron las tasas de morbilidad y se consideró el total de casos en menores de 0 a 4 años de edad, por ser este el grupo de población más vulnerable frente a las condiciones de saneamiento $(1,10)$. El denominador fue el número de habitantes para cada grupo tomado de los datos de la población reportados por el DANE para los años analizados. Para la enfermedad diarreica aguda, se consideró el número de casos por 1.000 habitantes y para las enfermedades transmitidas por alimentos y la hepatitis A, con menor número de casos reportados, el cálculo se hizo sobre 10.000 habitantes.

La mortalidad infantil se obtuvo en tasas calculadas para todos los municipios con la metodología establecida por el DANE (20). Los datos fueron sistematizados mediante Microsoft Excel-10 ${ }^{\mathrm{T}} \mathrm{M}$.

\section{Análisis de correlación}

Para analizar el comportamiento entre las variables de la calidad del agua y las enfermedades seleccionadas, se aplicaron pruebas de correlación, considerando el municipio como unidad de análisis. Para las variables de la calidad del agua, se utilizaron los valores medios de los resultados de los análisis de las muestras de agua de cada municipio por característica (color, pH, turbiedad, cloro residual líquido, coliformes totales, E. coli, y el índice del riesgo de la calidad del agua). Para las enfermedades consideradas se usaron las tasas de mortalidad infantil y las tasas de morbilidad por municipio. Las variables se evaluaron inicialmente para conocer la distribución de los datos analizados; para establecer la normalidad, se aplicó la prueba de Shapiro-Wilk, y para la homocedasticidad, la de Breusch-Pagan. El análisis se hizo mediante el cálculo del coeficiente de correlación de Spearman para datos no paramétricos con el programa SPSS $18^{\circledR}$ (PASW Statistics), considerando un nivel de significancia del $5 \%$.

\section{Mapa de riesgo}

El estudio también se propuso caracterizar mediante un mapa de riesgo las relaciones entre las variables de la calidad del agua y los eventos en salud, con el fin de identificar las zonas de mayor riesgo. Para elaborarlo, se recurrió al análisis de componentes principales, que permite transformar un grupo de variables en un nuevo conjunto de variables de menor tamaño, establecer su interrelación y expresarlas al final como una sola variable. Para la elaboración del mapa, se consideró la matriz de correlación de la calidad del agua indicada por el índice del riesgo de la calidad del agua y las tasas de mortalidad infantil consolidadas para el periodo 2008-2011, por presentar el mayor grado de correlación.

El análisis de componentes principales se hizo mediante el programa SPSSTM, Statistic Data Editor; para evaluar la aplicabilidad del análisis factorial, se utilizaron el test estadístico de KaiserMeyer-Olkin (KMO) y el test de Bartlett. El valor del KMO fue de 0,500 y el del test de Bartlett fue 
de 137,22 , con $p \leq 0,000$, ya que el factor resultado (variable) explicó el $67,40 \%$ de la variabilidad total. Los resultados de los coeficientes factoriales para cada municipio con evidencia de relación entre los dos eventos, se clasificaron mediante el método de Quantine en tres niveles de riesgo: alto, medio y bajo, usando el programa ArcView GISTM, versión 9.3.

\section{Resultados}

\section{Calidad del agua}

El análisis de las características microbiológicas indicó que el porcentaje de las muestras que se ajustaban a los valores máximos permitidos para E. coli, variaron entre 72 y $77 \%$. Los resultados de los coliformes totales evidenciaron un menor número de muestras dentro del valor de referencia, que osciló entre 60 y $67 \%$ (figura 1a). Es importante recordar que la presencia de $E$. coli en las muestras de agua es indicativa de contaminación fecal, lo que expone a la población consumidora a peligros por la presencia de agentes patógenos. En el caso de la presencia de coliformes totales en el agua de abastecimiento, aunque esta no es una indicación directa de contaminación por agentes patógenos, sí puede ser señal de contaminación del agua causada por fallas en la integridad del sistema de distribución (rupturas, derrames, intermitencia en el abastecimiento o formación de biopelículas, entre otras).

El análisis de las características fisicoquímicas evidenció que se cumplía con los valores máximos permitidos en más del $80 \%$, con excepción del cloro residual líquido (figura 1b). El pH y la turbiedad presentaron un porcentaje de cumplimiento de los valores máximos permitidos por encima del $90 \%$. En cuanto al color, los porcentajes de cumplimiento fueron casi del $80 \%$, en tanto que para el cloro residual líquido no sobrepasaron el $70 \%$, lo que reveló la falta de este elemento en el agua de consumo. En 2012 se presentó una disminución de los porcentajes de muestras aceptables para la medición de todos los parámetros.

El análisis de la distribución de los resultados de la medición del cloro residual líquido, evidenció una tendencia al aumento en sus valores (expresada en los valores de la mediana, que aumentó de $0,57 \mathrm{mg} / \mathrm{L}$ en 2008 a 0,76 en 2012), lo que significa que hubo un aumento de su concentración en el agua; sin embargo, la mayoría de los valores estuvieron por debajo de 1,0 mg/L (figura 2a). Estos porcentajes anómalos de cloro residual líquido, podrían explicar la presencia de coliformes totales y $E$. coli en las muestras analizadas debido a la falta de este elemento o al poco tiempo de contacto en la optimización durante la etapa de desinfección. Aunque los valores máximos permitidos establecidos por la norma se encuentran entre 0,3 y $2,0 \mathrm{mg} / \mathrm{l}$, no siempre los valores de cloro residual líquido más elevados o ajustados al rango logran garantizar una desinfección del agua suficiente para asegurar la eliminación de los patógenos bacterianos, los parásitos y algunos virus en los diferentes tramos del sistema de suministro, especialmente, si no se consideran los resultados de las demás características evaluadas que pueden interferir en su actividad, específicamente la turbiedad y el color.
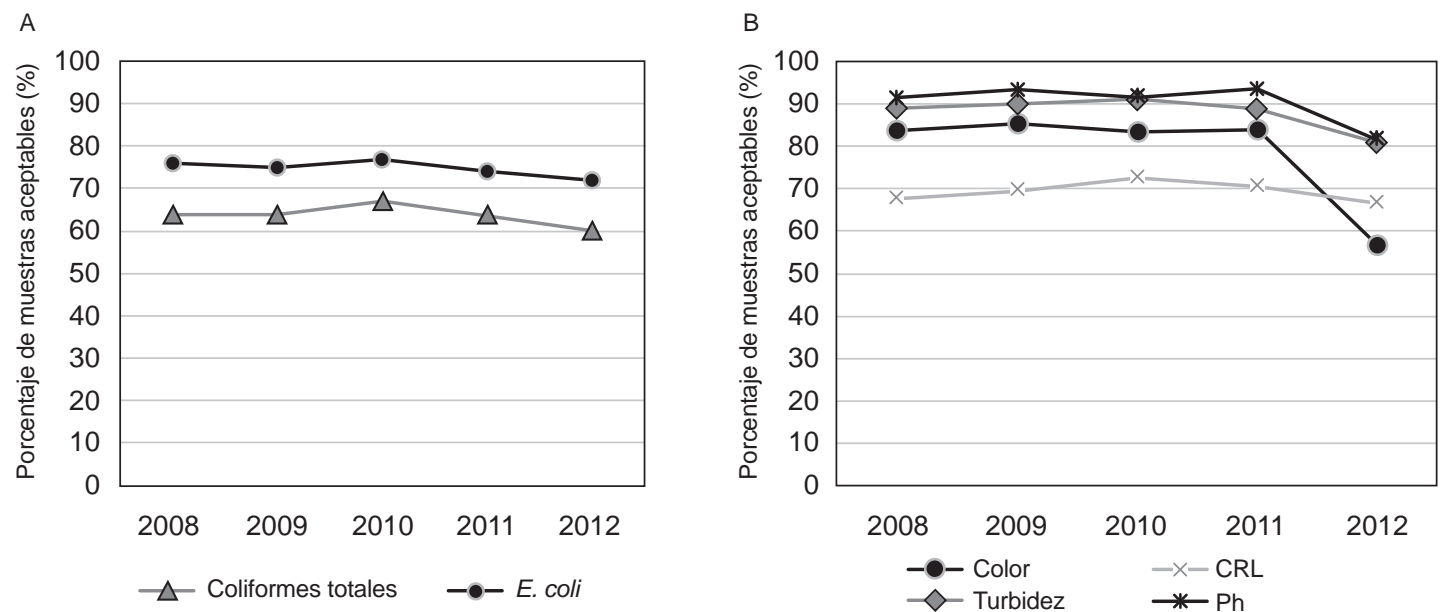

Figura 1. Porcentaje de aceptabilidad de las características microbiológicas y fisicoquímicas del agua, 2008-2012. A. Características microbiológicas. B. Características fisicoquímicas. CRL: cloro residual libre. 

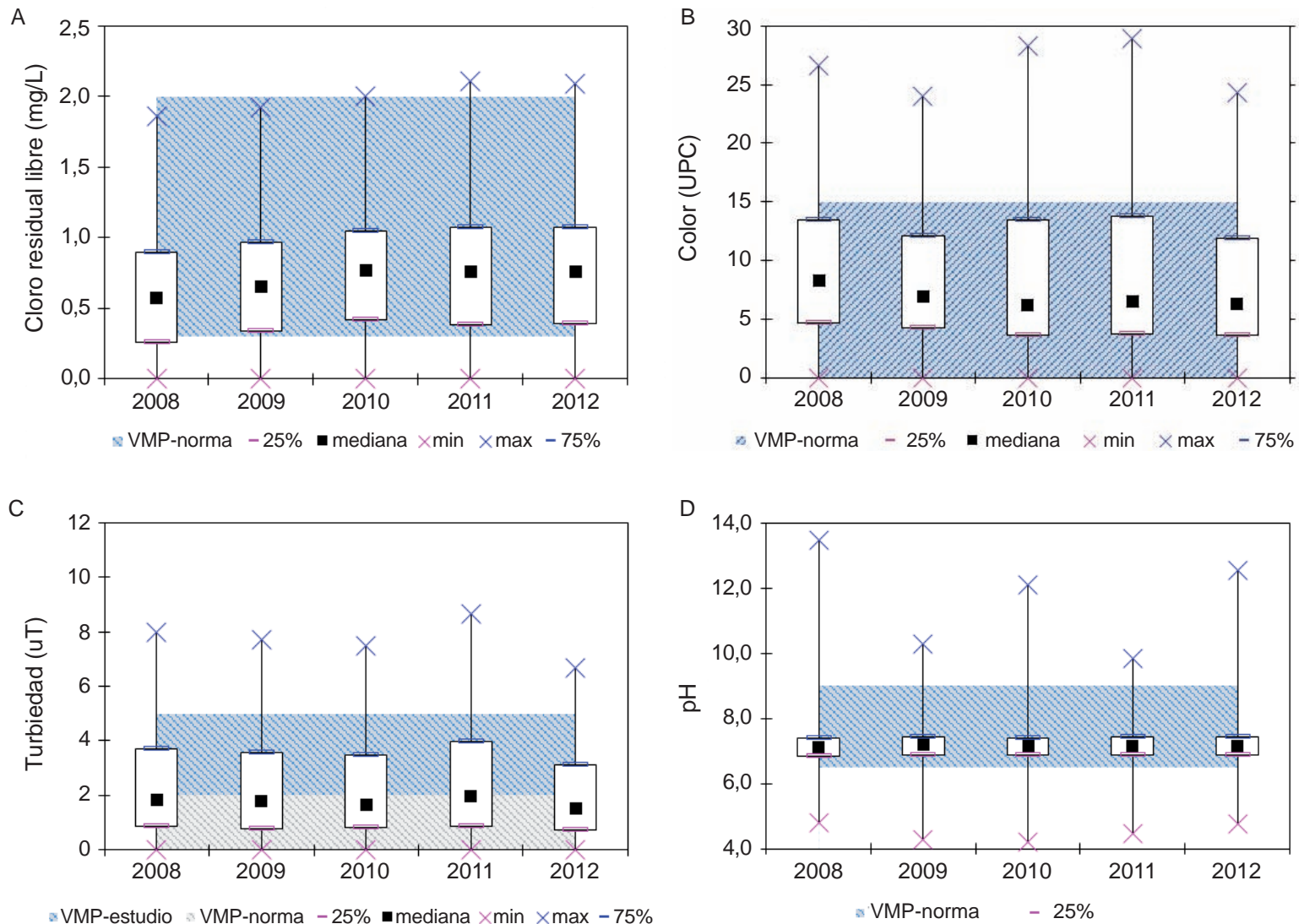

Figura 2. Estadística descriptiva de las características fisicoquímicas, Colombia, 2008-2012. A. Cloro residual libre. B. Color. C. Turbiedad. D. pH. VMP - norma: valor máximo permitido por la norma colombiana, VMP - estudio: valor máximo permitido considerado en este estudio

El color y la turbiedad son características relacionados con la carga de materia orgánica del agua, factor que influye notablemente en la eficiencia del tratamiento del agua, principalmente en la cloración. Los resultados de color revelaron una discreta tendencia a la disminución de los valores, expresada por la variación de 8,30 UPC en 2008 a 6,30 UPC en 2012. En este parámetro también se observó una amplia variación en la distribución de los datos a lo largo del período (desviación estándar en 2008: 27,09; en 2009: 24,93; en 2010: 24,03; en 2011: 29,37 y en 2012: 23,24 ) (figura $2 b$ ), y hubo muestras que presentaron valores máximos permitidos por encima de lo establecido en la legislación (15 UPC) en todos los años. De igual forma, los valores de turbiedad presentaron una gran variabilidad (desviación estándar en 2008: 6,49; en 2009: 7,02; en 2010: 7,12; en 2011: 8,29 y en 2012: 8,17), aunque en este parámetro la tendencia a lo largo del período fue estable (mediana de los valores en 2008: 1,82 UNT y en 2012: 1,54 UNT) (figura 2c).
Las variaciones en estas características están relacionadas con su sensibilidad frente a las modificaciones del ambiente, que para el color se refieren a la posible presencia de sustancias orgánicas y para la turbiedad, de material en suspensión (sólidos) como resultado de procesos de remoción poco eficientes en los sistemas de tratamiento de filtración por múltiples etapas, de filtración lenta en arena y de otros sistemas compactos, modulares o de tecnologías alternas de abastecimiento. Los valores elevados de color y turbiedad en el agua, comúnmente exigen un mayor contenido de cloro para la desinfección. La OMS, la Environmental Protection Agency (EPA) de los Estados Unidos y las guías de calidad del agua de Canadá, sugieren 0,1 UNT como valor ideal de la turbiedad del agua para una desinfección eficiente $(13,21,22)$. Cabe mencionar que estas normas permiten valores hasta de 1,0 UNT, dependiendo de que el sistema de tratamiento sea de filtración rápida o lenta. 
Los resultados del $\mathrm{pH}$ evidenciaron porcentajes de cumplimiento por encima del $90 \%$, con excepción de los correspondientes al año 2012. Los datos estadísticos revelaron poca variabilidad de los resultados (desviación estándar en 2008: 0,60; en 2009: 0,60; en 2010: 0,50; en 2011: 0,42 y en 2012: 0,48), en tanto que las medianas fueron de alrededor de 7,1 en todo el periodo (figura 2d). La normativa contempla un rango amplio de $\mathrm{pH}$ (entre $6,5$ y 9,0$)$; sin embargo, los valores estuvieron entre 6,5 y 7,5 , rango recomendado para una mayor disponibilidad de la especie activa de cloro residual, lo cual es un aspecto favorable para el proceso de desinfección y para mejorar la calidad del agua.

Es necesario aclarar que, aunque en la norma se establecieron valores máximos permitidos de hasta 2 UNT para la turbiedad, se estableció también un período de implementación de cinco años a partir de su publicación, con el fin de permitir la adecuación de las instalaciones para el cumplimiento del valor por parte de los sistemas de abastecimiento. Por lo tanto, en este estudio se consideró como valor máximo permitido de turbiedad 5 UNT, rango que es amplio, por lo que puede existir riesgo por la presencia de microrganismos como Cryptosporidium spp. y Giardia spp. Considerando un valor de 2 UNT como valor máximo permitido, el análisis de los resultados demostró un aumento del porcentaje de muestras que no cumplían con los estándares de potabilidad, que varió de 14 a $17 \%$ en el periodo, evidenciando un mayor deterioro de la calidad del agua. Los valores elevados observados para el color y la turbidez, pudieron disminuir las concentraciones de cloro residual líquido y, consecuentemente, propiciar la presencia de coliformes totales y E. coli, lo cual debe considerarse en el proceso de tratamiento y en la evaluación de la calidad del agua.

\section{Índice del riesgo de la calidad del agua}

En el período revisado se calculó el índice en 211.407 muestras; la clasificación de las muestras reportadas en todo el período, evidenció que $57,7 \%$ de ellas no presentaban riesgo, $1,5 \%$ se clasificaron como de riesgo bajo, 22,4 \% como de riesgo medio, y aproximadamente el $30 \%$ de ellas estaba en los niveles críticos: $22,4 \%$ presentaba riesgo alto y $7,8 \%$ eran sanitariamente inviables, lo que implica una mayor probabilidad de enfermedades transmitidas por el agua en la población consumidora. El índice de riesgo de la calidad del agua general entre 2008 y 2012, reveló valores entre 22,1 y $27,3 \%$, es decir, un nivel de riesgo medio que, conforme a la clasificación establecida, comprende el rango entre 14,1 y $35 \%$. Al final del periodo de estudio, se observó un aumento en este índice, que pasó de 23,8 a $27,3 \%$, evidenciando un deterioro de la calidad del agua, aunque se mantuvo el mismo nivel de riesgo (figura 3), lo que coincide con el análisis de cumplimento de los valores máximos permitidos establecidos en la legislación.

Los valores del índice del riesgo de la calidad del agua según la zona de recolección, presentaron grandes diferencias entre la zona rural y la urbana. En la zona urbana la calidad del agua distribuida fue mejor, con valores entre 11,5 y $16,7 \%$, es decir, niveles correspondientes a bajo y medio; en la zona rural, los valores se ubicaron en el nivel de riesgo alto, entre 47,8 y 49,7\%. En la zona urbana se observó una mejoría paulatina de la calidad del agua entre los años 2008 y 2011, con una leve desmejora en el 2012, tal como se observó en el índice del riesgo de la calidad del agua general, mientras que en la zona rural se vio un aumento de los valores de este índice, lo que denotaba un deterioro de la calidad del agua.

Los índices del riesgo de la calidad del agua calculados para 1.067 municipios de un total de 1.102 (96,8 \%) (figura 4), demostraron que en 195 de ellos (18,3\%), la calidad del agua correspondió al nivel sin riesgo y 370 (34,7\%), a niveles de riesgo alto y de inviabilidad sanitaria. Los resultados revelaron un alto porcentaje de municipios con agua de calidad inadecuada para el consumo humano. En la zona urbana, se analizaron 1.066 (96,7\%) municipios, de los cuales 295 (27,7\%) tenían mejor calidad del agua, pues esta no mostraba niveles de riesgo, en tanto que en 221 municipios (20,7\%) se registraron índices del riesgo de la calidad de agua, altos o de inviabilidad sanitaria. Aunque el porcentaje de municipios en el nivel sin riesgo aumentó, el porcentaje de aquellos con deterioro de la calidad fue significativo, considerando el impacto negativo que esto tiene, principalmente en los sistemas de abastecimiento urbano que suministran agua a un gran número de personas. En la zona rural, solamente 825 municipios (74,9\%) reportaron información sobre la calidad del agua, lo que demuestra la falta de vigilancia y dificulta, a su vez, el conocimiento real de las condiciones del agua y la adopción de acciones oportunas para su mejoramiento. En 79 municipios $(9,6 \%)$ de la zona rural, se encontraron buenas condiciones del agua 


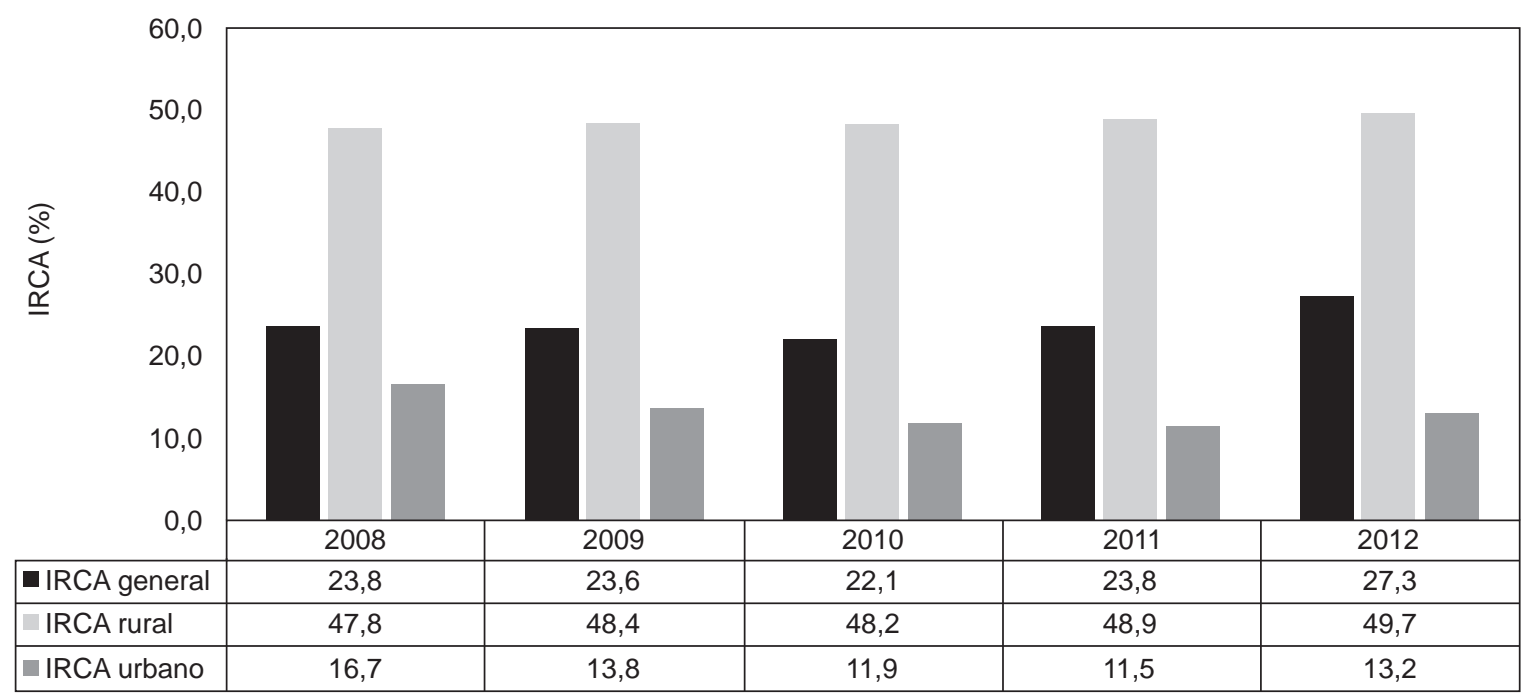

Figura 3. Distribución de los valores del índice del riesgo de la calidad del agua general, y para las áreas urbanas y rurales en Colombia, 2008-2012

IRCA: índice del riesgo de la calidad del agua

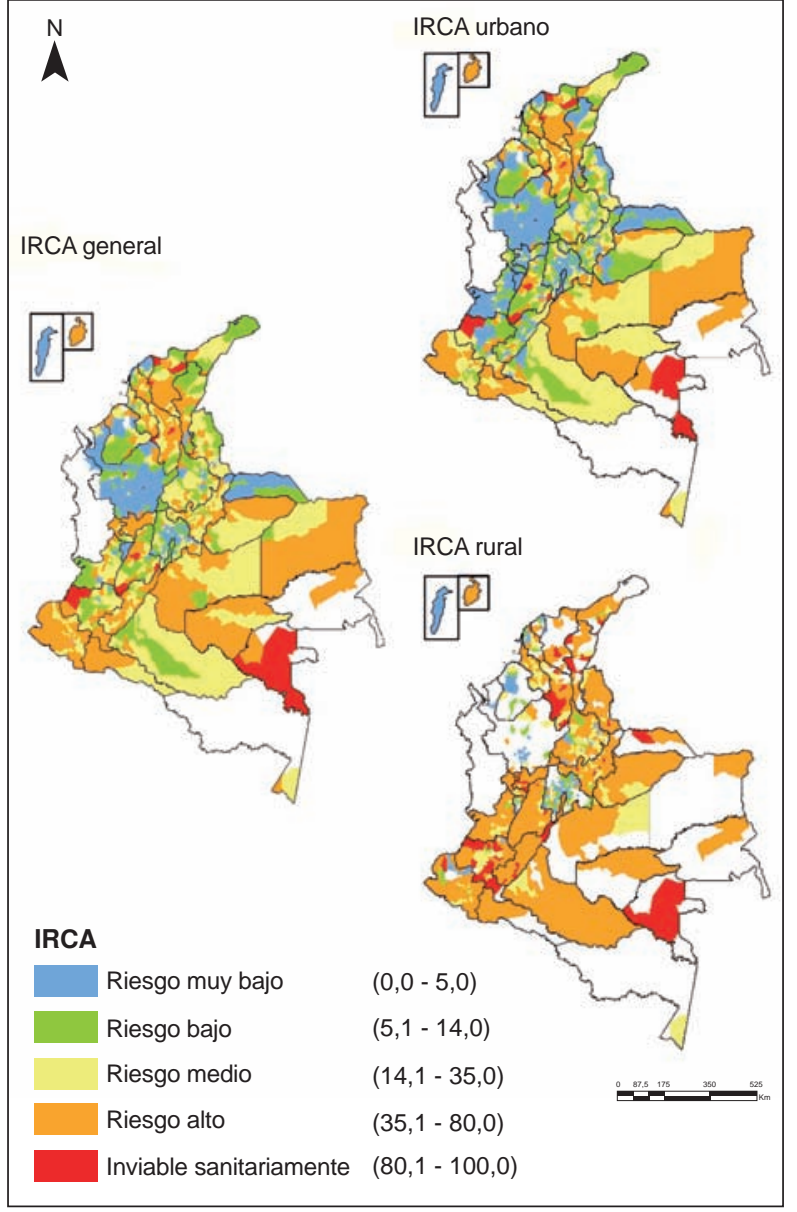

Figura 4. Distribución de la clasificación del índice del riesgo de la calidad del agua (IRCA) por municipios y por zona rural o urbana, Colombia, 2008-2012 para consumo (nivel sin riesgo), en tanto que en $565(68,5 \%)$ se presentaron niveles de riesgo alto o de inviabilidad sanitaria.

\section{Análisis de correlación entre la calidad del agua y la morbimortalidad}

Los resultados de los análisis de correlación entre la calidad del agua y la morbimortalidad, se presentan en la cuadro 1. Se observó una mayor correlación entre la mortalidad infantil y la calidad del agua durante los cuatro años estudiados. La mortalidad infantil mostró una correlación positiva con los valores elevados encontrados en los parámetros de color, turbiedad, coliformes totales, E. coli y el índice del riesgo de la calidad del agua. Las características de $\mathrm{pH}$ y cloro residual líquido tuvieron una correlación inversa, es decir que los valores bajos de $\mathrm{pH}$ y la disminución en la concentración de cloro residual líquido se relacionaron con el aumento de la mortalidad infantil. El índice de riesgo de la calidad de agua tuvo los valores más altos de correlación, lo que indica una asociación significativa entre la poca calidad del agua y la mortalidad infantil.

En los resultados del análisis de correlación entre las características de la calidad del agua y la enfermedad diarreica aguda se identificaron algunos valores de correlación positiva; sin embargo, no se observó una continuidad en los años subsiguientes. En algunos casos se presentaron correlaciones inversas, como sucedió con el cloro residual líquido, la turbiedad y el índice del riesgo de la calidad del 
Cuadro 1. Resultados del análisis de correlación entre las enfermedades seleccionadas y la calidad del agua, 2008-2012

\begin{tabular}{|c|c|c|c|c|c|c|c|c|c|c|}
\hline \multirow[t]{2}{*}{ Parámetros } & \multicolumn{5}{|c|}{ Mortalidad infantil ${ }^{1}$} & \multicolumn{5}{|c|}{ EDA $^{2}$} \\
\hline & 2008 & 2009 & 2010 & 2011 & 2012 & 2008 & 2009 & 2010 & 2011 & 2012 \\
\hline Color & 0,015 & 0,021 & $0,080^{*}$ & $0,045^{\star}$ & & $-0,097^{*}$ & $-0,065$ & $-0,073$ & $-0,036$ & $-0,001$ \\
\hline Turbiedad & $0,141^{*}$ & $0,114^{*}$ & $0,126^{*}$ & 0,068 & & $0,103^{*}$ & $-0,030$ & $-0,044$ & $-0,078^{*}$ & 0,007 \\
\hline $\mathrm{pH}$ & $-0,099^{*}$ & $-0,084^{*}$ & $-0,095^{*}$ & $0,030^{*}$ & & 0,045 & $-0,026$ & $-0,056$ & 0,012 & $-0,008$ \\
\hline Cloro residual líquido & $-0,253^{*}$ & $-0,197^{*}$ & $-0,197^{*}$ & $-0,170^{*}$ & & $-0,093^{*}$ & $-0,001$ & $0,123^{*}$ & $-0,045$ & $-0,023$ \\
\hline Coliformes totales & $0,192^{*}$ & $0,154^{*}$ & $0,136^{*}$ & 0,060 & & $0,107^{*}$ & $-0,014$ & 0,046 & $-0,032$ & $-0,019$ \\
\hline Escherichia coli & $0,172^{*}$ & $0,141^{*}$ & $0,120^{*}$ & 0,047 & & $0,168^{*}$ & $-0,024$ & $0,085^{\star}$ & $-0,022$ & $-0,023$ \\
\hline $\begin{array}{l}\text { Índice del riesgo de la } \\
\text { calidad de agua }\end{array}$ & $0,382^{*}$ & $0,310^{*}$ & $0,307^{*}$ & $0,268^{*}$ & & 0,055 & 0,064 & $-0,096^{*}$ & 0,030 & $-0,016$ \\
\hline \multirow[t]{2}{*}{ Parámetros } & \multicolumn{5}{|c|}{ EDA en menores 0 a 4 años ${ }^{3}$} & \multicolumn{5}{|c|}{ ETA $^{4}$} \\
\hline & 2008 & 2009 & 2010 & 2011 & 2012 & 2008 & 2009 & 2010 & 2011 & 2012 \\
\hline Color & $0,095^{*}$ & 0,027 & $0,110^{*}$ & $-0,080$ & 0,011 & 0,063 & 0,085 & 0,147 & $-0,081$ & 0,086 \\
\hline Turbiedad & $0,172^{*}$ & 0,069 & 0,088 & 0,198 & 0,011 & 0,238 & 0,063 & 0,099 & 0,039 & 0,000 \\
\hline $\mathrm{pH}$ & $-0,009$ & 0,013 & $-0,006$ & $-0,010$ & 0,032 & $-0,025$ & 0,041 & $-0,034$ & $-0,014$ & $-0,041$ \\
\hline Cloro residual líquido & $-0,069$ & $-0,002$ & $-0,020$ & $-0,213$ & $-0,073$ & 0,020 & $-0,132$ & $-0,002$ & 0,072 & $-0,094$ \\
\hline Coliformes totales & $0,125^{\star}$ & $0,115^{*}$ & 0,041 & 0,230 & $0,086^{*}$ & 0,150 & 0,052 & 0,117 & $-0,002$ & 0,050 \\
\hline Escherichia coli & $0,106^{*}$ & 0,067 & $0,068^{*}$ & 0,081 & 0,074 & $0,162^{*}$ & 0,066 & 0,151 & $-0,094$ & $-0,011$ \\
\hline $\begin{array}{l}\text { Índice del riesgo de la } \\
\text { calidad de agua }\end{array}$ & $0,168^{*}$ & 0,087 & $0,071^{*}$ & $0,326^{*}$ & 0,074 & 0,148 & 0,120 & 0,170 & 0,025 & 0,110 \\
\hline \multirow[t]{2}{*}{ Parámetros } & \multicolumn{5}{|c|}{ ETA en menores 0 a $4^{5}$} & \multicolumn{5}{|c|}{ Hepatitis $A^{6}$} \\
\hline & 2008 & 2009 & 2010 & 2011 & 2012 & 2008 & 2009 & 2010 & 2011 & 2012 \\
\hline Color & 0,014 & 0,001 & $-0,033$ & $-0,080$ & 0,139 & 0,049 & 0,030 & $0,117^{*}$ & $-0,035$ & 0,106 \\
\hline Turbiedad & 0,083 & 0,021 & $-0,080$ & 0,198 & 0,034 & 0,081 & 0,012 & $0,088^{*}$ & 0,015 & 0,070 \\
\hline $\mathrm{pH}$ & 0,014 & 0,164 & 0,091 & $-0,010$ & 0,041 & $0,090^{*}$ & 0,011 & $-0,033$ & $-0,093^{*}$ & $-0,148^{*}$ \\
\hline Cloro residual líquido & $-0,094$ & $-0,223$ & 0,123 & $-0,213$ & $-0,126$ & $-0,041$ & $-0,065$ & $-0,002$ & $-0,111^{*}$ & $-0,231^{*}$ \\
\hline Coliformes totales & 0,143 & 0,080 & $-0,059$ & 0,230 & 0,024 & $0,103^{*}$ & $-0,065$ & $-0,004$ & $-0,041$ & $-0,006$ \\
\hline Escherichia coli & 0,125 & $-0,004$ & $-0,050$ & 0,081 & $-0,031$ & $0,090^{*}$ & $-0,027$ & 0,064 & 0,028 & 0,032 \\
\hline $\begin{array}{l}\text { Índice del riesgo de la } \\
\text { calidad de agua }\end{array}$ & 0,211 & 0,187 & $-0,010$ & 0,326 & 0,155 & 0,073 & 0,029 & 0,052 & $0,171^{*}$ & $0,204^{*}$ \\
\hline
\end{tabular}

* Resultados del análisis de correlación mediante el coeficiente de correlación de Spearman, significancia estadística a<0,05

${ }^{1}$ : tasa de mortalidad infantil por $1.000 ;{ }^{2}$ : tasa de morbilidad por enfermedad diarreica aguda (EDA) por $1.000,{ }^{3}$; tasa de morbilidad por enfermedad diarreica aguda en menores de 0 a 4 años por 1.000; ${ }^{4}$ : tasa de morbilidad general por enfermedades transmitidas por alimentos (ETA) por 10.000; 5: tasa de morbilidad por enfermedades transmitidas por alimentos en menores de 0 a 4 años por $10.000 ;{ }^{6}$ : tasa de morbilidad por hepatitis A por 10.000

agua, lo que evidenció comportamientos diferentes a los esperados: disminución de la enfermedad diarreica aguda al disminuir las concentraciones de cloro residual líquido o aumento de la turbiedad y el índice del riesgo de la calidad del agua. No hubo correlación positiva entre los parámetros de calidad del agua y la tasa de morbilidad por enfermedad diarreica aguda, en tanto que la hepatitis A presentó correlación positiva con algunas de las características de la calidad del agua, evidenciando un comportamiento más estable, lo que explica mejor la relación entre la calidad del agua y la salud.

\section{Mapa de riesgo}

En el mapa de riesgo se reflejó la relación entre la calidad del agua y la mortalidad infantil en los municipios (figura 5). Se consideraron los resultados de los análisis de correlación y los test factoriales, lo que permitió una apreciación más objetiva de la relación de los dos eventos. Los niveles de riesgo hallados permitieron concluir que la mayoría de los municipios en el nivel de riesgo bajo, pertenecían a los departamentos de Antioquia (76,0\%), Atlántico $(69,6 \%)$, Cundinamarca $(74,1 \%)$, Norte de Santander $(57,5 \%)$ Quindío (100\%) y Bogotá, (100\%), los cuales están ubicados en la región central y nororiental del país. Los municipios con niveles altos de riesgo y de mortalidad infantil, así como en el índice de riesgo de la calidad del agua, pertenecían a los departamentos de Amazonas (100\%), Bolívar (56,5\%), Caldas (70,4 \%), Casanare (78,9 \%) Guainía (100\%), Guaviare (100\%), Magdalena (66,7 \%), Meta (86,2 \%) Nariño $(60,9 \%)$, Putumayo $(61,5 \%)$ Vaupés $(100 \%)$ y 


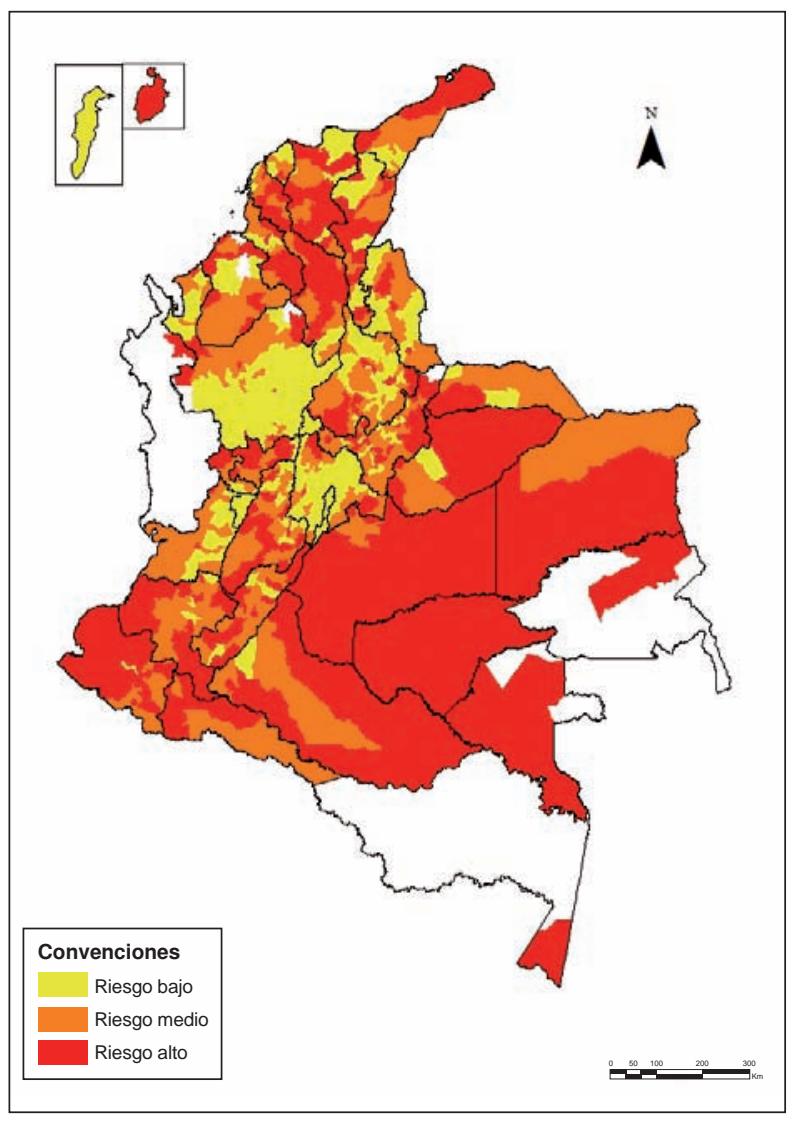

Figura 5. Distribucion de los municipios según la clasificación del riesgo de la calidad del agua (IRCA) y la mortalidad infantil, 2008-2011

Vichada (50\%), localizados principalmente en la región amazónica, ubicándose los otros en la Orinoquia, el Caribe, el Pacífico y la región central del país. Cabe resaltar la ausencia de datos de los municipios del Chocó, departamento que no notificó la información sobre la calidad del agua en esos años.

\section{Discusión}

El estudio de las características microbiológicas, físicas y químicas en el marco de la vigilancia sanitaria, permitió avanzar en la identificación de los problemas de la calidad del agua en el país, información valiosa para la búsqueda de soluciones que permitan mejorar los sistemas de abastecimiento y aumentar el acceso al agua potable. Es importante resaltar que los resultados analizados corresponden a una gran diversidad de regiones, territorios y sistemas de tratamiento, cuyas particularidades dificultan el análisis minucioso de los problemas de la calidad del agua distribuida; sin embargo, los hallazgos permitieron explorar de forma general la situación de la calidad del agua en Colombia y su potabilidad con un enfoque diferencial.

El análisis de los resultados indica que existe un alto porcentaje de municipios que distribuyen agua que no se ajusta a los estándares de potabilidad. El estudio de las características reveló un alto porcentaje de muestras con coliformes totales y $E$. coli que excedían los valores máximos permitidos de potabilidad, lo que indica la posible presencia de microorganismos patógenos en el agua suministrada. También, fue notable el alto porcentaje de muestras que no se ajustaban a los estándares de cloro residual líquido, lo que puede asociarse con las características microbiológicas del agua, por lo que debería optimizarse la etapa de desinfección del agua en los sistemas de abastecimiento.

Se demostró la relevancia de las características de turbiedad y color para el análisis de la calidad del agua y, aunque la mayoría de los resultados se mantuvieron dentro de los valores máximos permitidos de potabilidad, se evidenció un amplio rango de dispersión, así como variabilidad en los datos y altos valores. Esta variabilidad de los valores de turbiedad y de color, podría tener relación con la eficacia de la desinfección, aumentando la cantidad necesaria de cloro residual líquido, aspecto que debe considerarse en los procesos de tratamiento. En lo referente a la turbiedad, el cambio de 5 UNT a 2 UNT en los valores máximos permitidos, puede mejorar notablemente la calidad del agua; no obstante, sería importante que la norma contemplase valores por debajo de 1,0 UNT, que es la medida que se maneja actualmente en la mayoría de países para disminuir los riesgos para la salud, aunque se requieren inversiones para desarrollar los procesos de tratamiento que permitan cumplir con estas exigencias.

A lo largo del período no se identificó un aumento en el porcentaje de muestras que cumplieran con los valores máximos permitidos establecidos en la legislación, lo que sugiere la necesidad de promover o reforzar acciones para mejorar la calidad del agua, en las cuales deberían participar, al menos, el sector de la salud y los servicios de abastecimiento del agua. Las autoridades de salud tienen un papel estratégico en el mejoramiento del agua de consumo; en primer lugar, deben recolectar la información sobre la calidad del agua y exigir el cumplimiento de los parámetros de potabilidad $y$, en segundo lugar, deben promover estrategias 
para el mejoramiento de la calidad. Asimismo, la información sobre la calidad del agua deberá complementarse con la información proveniente del sector de la salud, con el fin de desarrollar acciones que reduzcan los riesgos inherentes a un deficiente tratamiento del agua y eviten el surgimiento de posibles brotes de enfermedades de origen hídrico.

En la zona rural se encontraron los mayores problemas en términos de la calidad del agua; la población en estas zonas está expuesta a un gran riesgo de enfermedades transmitidas por el agua de consumo, lo cual acentúa las desigualdades en el acceso al agua potable y afecta las condiciones de salud. El mejoramiento de la calidad del agua en estas zonas constituye un reto en la formulación de políticas públicas orientadas a transformar la situación de riesgo y las vulnerabilidades identificadas para alcanzar un mejor acceso al agua potable de calidad. Además del deterioro de la calidad del agua, en algunos municipios no se reportó la información sobre la calidad en la zona rural, lo cual impide conocer la situación real y, consecuentemente, adoptar estrategias de intervención. La vigilancia de la calidad del agua debe ser universal y responder a una perspectiva global del territorio que considere a todos los grupos de la población, sin exclusión, con el fin de detectar los problemas prioritarios, las carencias de los diversos segmentos sociales y los riesgos desiguales en el ejercicio de una gestión más equilibrada y equitativa del recurso (23).

Los resultados apuntan a que es indispensable fortalecer los sistemas de abastecimiento, para aumentar la eficiencia en la remoción de contaminantes y reducir los riesgos para la salud. La eficiencia de los sistemas de abastecimiento se alcanza mediante compromisos políticos, sociales y económicos $(24,25)$. En países en desarrollo se ha evidenciado que la falta de apoyo político, el uso inadecuado o incorrecto de los fondos, la mala gestión y la no recuperación de costos, han producido deficiencias en el sistema de distribución, así como en su operación, en tanto que la falta de mantenimiento ha llevado al colapso de los sistemas de abastecimiento. Esto se debe a que la provisión de un nuevo sistema de suministro de agua para una comunidad depende muchas veces de los réditos que esto aporte a las campañas políticas, mientras que su operación y mantenimiento pasan a tener menor relevancia (26). Ante esta problemática, es esencial el fortalecimiento de la gobernanza en salud ambiental en los territorios para movilizar los recursos políticos e institucionales a favor del mejoramiento del agua, con la participación de la comunidad y de todos los sectores vinculados (27).

Los resultados indicaron una correlación significativa de la calidad del agua con la mortalidad infantil, lo que se evidencia en el hecho de que los territorios con deficiencias en la calidad del agua para consumo humano presentaron un aumento de las tasas de mortalidad infantil. Algunos estudios regionales han obtenido resultados similares a los observados; en uno de ellos, basado en datos secundarios de diferentes países latinoamericanos y del Caribe, se confirmó la asociación entre la tasa de mortalidad infantil y la cobertura en el abastecimiento del agua, y se concluyó que una infraestructura sanitaria adecuada en los países con condiciones deficientes de saneamiento ambiental podría reducir la mortalidad infantil y la mortalidad en menores de cinco años de edad (28). En Brasil, donde se analizó la asociación entre la mortalidad infantil en menores de cinco años debida a enfermedades de origen hídrico y el saneamiento ambiental en las microrregiones del país, se observó una relación directa entre el saneamiento inadecuado en la vivienda y la mortalidad en menores de cinco años debida a enfermedades de transmisión; asimismo, se identificaron mayores riesgos para la salud relacionados con el saneamiento inapropiado en microrregiones con grandes concentraciones de población en condición de pobreza y con poca educación (29).

La asociación positiva de la mortalidad infantil y la calidad del agua en comparación con los demás eventos propuestos en este estudio, puede ser resultado de la metodología empleada para la estimación de la mortalidad infantil, ya que esta se hace mediante la explotación intensiva de fuentes de estadísticas vitales, e incluye el análisis del índice de años de vida perdidos con el cual se miden el nivel y los cambios en la mortalidad infantil, por lo que constituye un indicador más confiable (20).

Con respecto a los demás eventos seleccionados, a pesar de ser bastante reconocidos por su sensibilidad frente a las condiciones del ambiente y del saneamiento, como lo revelan diferentes estudios en el caso de la enfermedad diarreica aguda (2-4), esta no demostró claramente una correlación positiva con las características de la calidad del agua. La falta de correlación entre la calidad del agua y la enfermedad diarreica aguda, las enfermedades transmitidas por alimentos y 
la hepatitis $A$, puede responder a las debilidades en la notificación de los eventos, a los problemas de subregistro y a la falta de calidad de los datos reportados, lo cual dificulta la estimación de la incidencia real de las enfermedades. Un aspecto importante en el reporte de estas enfermedades es la ausencia de una cultura de búsqueda de la atención médica para los casos de diarrea por parte de la población.

El informe sobre la vigilancia de la enfermedad diarreica aguda evidenció un posible subregistro de los casos de morbilidad, así como deficiencias en la oportunidad del reporte y poca calidad de los datos. Se observaron incongruencias en el número de casos notificados por grupos de edad, por sexo, y por estado vivo o muerto del paciente, entre otras variables; en algunos casos la semana no correspondía a las fechas de notificación, y había errores de digitación y de ajuste de casos (30). En cuanto a las enfermedades transmitidas por alimentos, se observó una notificación deficiente de los brotes al Sivigila, así como demoras en la entrega de los resultados por parte de los laboratorios de salud pública departamentales debidas a las dificultades en el manejo del programa informático en las unidades primarias generadores de datos (31). En lo concerniente a la hepatitis A, se identificaron departamentos silenciosos, que no notifican los casos, así como datos de mala calidad y deficiencias en el registro de hallazgos semiológicos y datos específicos, así como del seguimiento en el municipio (32). En consecuencia, la falta de notificación es una limitación importante para el conocimiento de la incidencia de los eventos en salud en Colombia, lo que dificulta la integración de las estadísticas sobre salud y ambiente en el país. Estas limitaciones pueden tener relación con el incumplimiento por parte de las autoridades de las medidas necesidades de la vigilancia detectadas en el estudio de López, et al. (33).

Por otro lado, la evaluación del sistema de vigilancia de la calidad del agua para consumo humano, reflejó deficiencias y diferencias en su implementación en los territorios, pues en algunos de ellos el proceso está más avanzado en términos de ejecución de acciones; sin embargo, se identificaron problemas en cuanto a la universalidad de dichas acciones y la disponibilidad de recursos humanos, materiales y de infraestructura, aspectos que pueden afectar la vigilancia de la calidad del agua y la representatividad de las muestras, lo cual debe considerarse en el análisis (34).
Los estudios ecológicos son de gran relevancia en la aproximación a la situación de grandes poblaciones, pues posibilita la comparación entre regiones, la identificación de las áreas más críticas y los factores que deben ser objeto prioritario de intervenciones; además, presentan ventajas concretas en términos de la facilidad de ejecución, ya que se basan en datos secundarios, lo que repercute en el bajo costo de implementación. Por el otro lado, tienen poco poder analítico en comparación con otros tipos de estudios epidemiológicos y no alcanzan la sensibilidad necesaria para identificar la asociación entre la calidad del agua y las tres enfermedades aquí seleccionadas.

Los mapas de riesgo son instrumentos cartográficos ampliamente utilizados en salud pública porque permiten evidenciar la distribución geográfica de las exposiciones, los factores de riesgo y los agentes etiológicos. El mapa de riesgo elaborado en el presente estudio demostró su eficiencia para representar la relación entre la calidad del agua y la mortalidad infantil, evidenciando los municipios que presentaban mayor riesgo. De todas maneras, es importante considerar las limitaciones de las bases de datos primarias, lo que pudo influir en los bajos coeficientes de correlación, por lo que se recomienda fortalecerlas para mejorar la veracidad de los mapas.

El estudio de las relaciones entre la calidad del agua y la salud constituye una herramienta importante, para la adopción de decisiones en los diferentes sectores y el apoyo a las acciones de mejoramiento de la calidad del agua y, por ende, de la calidad de vida de la población. Las dificultades para estimar la carga de morbilidad atribuible al medio ambiente detectadas en Colombia, también se han identificado en toda la región de América Latina, principalmente las relacionadas con la falta de capacidad técnica y científica en la recolección de los datos iniciales sobre la mortalidad y la morbilidad, lo que ha dado lugar a errores de clasificación y a la notificación incompleta de las exposiciones ambientales (35).

En este sentido, es vital el fortalecimiento de los programas de vigilancia en salud ambiental para obtener información oportuna, sistemática y de calidad que permita conocer fielmente las relaciones entre la salud y el ambiente. El fortalecimiento de los sistemas de vigilancia debe ir acompañado del establecimiento de marcos políticos e institucionales que respalden la implementación de estos 
programas, así como del desarrollo de tecnologías apropiadas para los diferentes contextos $(35,36)$. Es fundamental, igualmente, el desarrollo del recurso humano, el aumento de la capacidad analítica de los laboratorios y el fortalecimiento de los procesos de gobernanza, en aras de una ejecución satisfactoria de las acciones de vigilancia.

\section{Agradecimientos}

Agradecemos a las secretarías de salud departamentales y municipales y a la Red de Laboratorios de Salud Pública encargada de las acciones de vigilancia de la calidad del agua para consumo humano en el país; a la Oficina de Tecnologías de la Información y las Comunicaciones y al Grupo de Calidad de Agua, así como a los referentes del sistema de información SIVICAP, a la Dirección de Salud Pública, al sistema de información Sivigila, a la Dirección de Redes en Salud Pública y al Observatorio Nacional de Salud del Instituto Nacional de Salud por el apoyo en la elaboración del documento.

\section{Conflicto de intereses}

Los autores manifestamos no tener conflictos de intereses con respecto a esta publicación.

\section{Financiación}

Este estudio fue financiado por el Instituto Nacional de Salud.

\section{Referencias}

1. Prüss-Üstün A, Bos R, Gore F, Bartram J. Safer water, better health: Costs, benefits and sustainability of interventions to protect and promote health. Geneva: World Health Organization; 2008.

2. Vázquez ML, Mosquera M, Cuevas LE, González ES, Veras ICL, Luz EO, et al. Incidência e fatores de risco de diarréia e infecções respiratórias agudas em comunidades urbanas de Pernambuco, Brasil. Cad Saúde Pública. 1999;15:163 72. http://dx.doi.org/10.1590/S0102-311X1999000100016

3. Heller L, Colosimo EA, Antunes CM. Environmental sanitation conditions and health impact: A case-control study. Rev Soc Bras Med. 2003;36:41-50. http://dx.doi. org/10.1590/S0037-86822003000100007

4. Ferrer DR, Strina A, Sandra RJ, Ribeiro HC, Cairncross S, Rodrigues IC, et al. A hierarchical model for studying risk factors for childhood diarrhoea: A case-control study in a middle-income country. Int J Epidemiol. 2008;37:805-15. http://dx.doi.org/10.1093/ije/dyn093

5. Esrey SA, Potash JB, Schiff C. Effects of improved water supply and sanitation on ascariasis, diarrhea, dracunculiasis, hookworm infection, schistosomiasis, and trachoma. Bull World Health Organ. 1991;69:609-13.

6. Baltazar JC, Nadera DP, Victora CG. Evaluation of the National Control of Diarrhoeal Disease Programme in the
Philippines, 1980-93. Bull World Health Organ. 2002;80:63743. http://dx.doi.org/10.1590/S0042-96862002000800008

7. Fewtrell L, Kaufmann RB, Kay D, Enanoria W, Haller L, Colford JM. Water, sanitation, and hygiene interventions to reduce diarrhoea in less developed countries: A systematic review and meta-analysis. Lancet Infect Dis. 2005;5:42-52. http://dx.doi.org/10.1016/S1473-3099(04)01253-8

8. Barreto ML, Genser B, Strina A, Teixeira MG, Assis AM, Rego RF, et al. Effect of city-wide sanitation programme on reduction in rate of childhood diarrhoea in northeast Brazil: Assessment by two cohort studies. Lancet. 2007;370:1622-8. http://dx.doi.org/10.1016/S0140-6736(07)61638-9

9. Cairncross S, Hunt C, Boisson S, Bostoen K, Curtis V, Fung IC, et al. Water, sanitation and hygiene for the prevention of diarrhea. Int J Epidemiol. 2010;39:193-205. http://dx.doi.org/10.1093/ije/dyq035

10. Prüss-Üstün A, Corvalán $\mathbf{C}$. Ambientes saludables y prevención de enfermedades: hacia una estimación de la carga de morbilidad atribuible al medio ambiente. Ginebra: Organización Mundial de la Salud; 2006.

11. World Health Organization. Progress on drinking-water and Sanitation-2012 Update. Geneva: World Health Organization; 2012.

12. Pan American Health Organization. The environment and human security. In: Health in the Americas. Washington, D.C.: PAHO; 2012.

13. World Health Organization. Guidelines for drinking water quality. Fourth edition. Geneva: World Health Organization; 2011.

14. Roja R. Guía para la vigilancia y control de la calidad del agua para consumo humano. Lima: Centro Panamericano de Ingeniería Sanitaria y Ciencia Ambiental/Organización Panamericana de la Salud; 2002.

15. Ministerio de la Protección Social. Decreto 1575 del 9 de mayo de 2007, por el cual se establece el Sistema para la Protección y Control de la Calidad del Agua para Consumo Humano. Diario Oficial 46623. Bogotá: Imprenta Nacional de Colombia; 2007. Fecha de consulta: 6 de abril de 2013. Disponible en: http://www.ins.gov.co/sivicap/Normatividad/ Decreto\%201575\%20de\%202007.pdf.

16. Ministerio de la Protección Social. Ministerio de Ambiente, Vivienda y Desarrollo Territorial. Resolución 2115 de 22 junio de 2007, por medio de la cual se señalan características, instrumentos básicos y frecuencias del sistema de control y vigilancia para la calidad del agua para consumo humano. Diario Oficial 46679. Bogotá: Imprenta Nacional de Colombia; 2007. Fecha de consulta: 6 de abril de 2013. Disponible en: http://www.ins.gov.co/sivicap/Normatividad/ Resoluci\%C3\%B3n\%202115\%20de\%202007.pdf.

17. Instituto Nacional de Salud. Guía del participante en el programa interlaboratorios de control de calidad de aguas potables - PICCAP. Bogotá: Instituto Nacional de Salud; 2014.

18. Grabow WOK. Water and Public Health. In: Knowledge for sustainable development. An insight into the Encyclopedia of Life Support Systems. Oxford, UK: UNESCO PublishingEolss Publishers; 2002.

19. Mara DD, Feachem RG. Water-and excreta-related diseases: Unitary environmental classification. J Environ Eng. 1999;125:334-9. http://dx.doi.org/10.1061/(ASCE)07339372(1999)125:4(334)) 
20. Departamento Administrativo Nacional de Estadísticas. Estimación del cambio en los niveles de la mortalidad infantil departamental y municipal a partir de las estadísticas vitales. Bogotá: Departamento Administrativo Nacional de Estadísticas; 2011.

21. United States Environmental Protection Agency. National Primary Drinking Water. Regulations: Long Term 2 Enhanced Surface Water Treatment Rule. Final Rule. EPA. Federal Register. 2006;71:654-786.

22. Federal Provincial Territorial Committee on Drinking Water. Guidelines for Canadian drinking water quality. Ottawa: Health Canada; 2008.

23. Monken M, Barcellos C. Vigilância em saúde e território utilizado: possibilidades teóricas e metodológicas. Cad Saúde Pública. 2005;21:898-906.

24. World Bank. Local solutions improve water supply and sanitation services in Colombia. Fecha de consulta: 28 de mayo de 2013. Disponible en: http://siteresources. worldbank.org/INTWSS/Resources/colombia.pdf.

25. World Health Organization. Global Water Supply and Sanitation Assessment 2000 Report. Iseman Creative, Washington, D.C.: World Health Organization; 2000.

26. World Health Organization. Constraints affecting the development of the water supply and sanitation sector. Geneva: World Health Organization; 2003.

27. Finkelman J, Galvao LA, Henao S. Governança da saúde ambiental na America Latina. Determinantes ambientais e sociais da saúde. In: Galvao LA, Finkelman J, Henao S, editores. Determinantes ambientais e sociais da saúde. Washington, D.C.: Organização Pan-Americana da Saúde/ Editora Fiocruz; 2011. p. 33-66.

28. Teixeira JC, Guilhermino RL. Análise da associação entre saneamento e saúde nos estados brasileiros, empregando dados secundários do banco de dados indicadores e dados básicos para a saúde 2003- IDB 2003. Eng Sanit Ambient. 2006;11:277-82. http://dx.doi.org/10.1590/S141341522006000300011
29. Bellido JG, Barcellos C, Barbosa FS, Bastos FI. Saneamiento ambiental y mortalidad en niños menores de 5 años por enfermedades de transmisión hídrica en Brasil. Rev Panam Salud Pública. 2010;28:114-20. http://dx.doi. org/10.1590/S1020-49892010000800007

30. Instituto Nacional de Salud. Informe del evento mortalidad por enfermedad diarreica aguda en menores de cinco años y morbilidad en todos los grupos de edad, hasta el periodo epidemiológico 6 del año 2014. Bogotá: Instituto Nacional de Salud; 2014.

31. Instituto Nacional de Salud. Informe del evento enfermedades transmitidas por alimentos, hasta el periodo epidemiológico VI del año 2014. Bogotá: Instituto Nacional de Salud; 2014.

32. Instituto Nacional de Salud. Informe del evento hepatitis A, hasta el quinto periodo epidemiológico del año 2014. Bogotá: Instituto Nacional de Salud; 2014.

33. López YL, González C, Gallego BN, Moreno AL. Rectoría de la vigilancia en salud pública en el sistema de seguridad social en salud de Colombia: estudio de casos. Biomédica. 2009;29:567-581. http://dx.doi.org/10.7705/ biomedica.v29i4.134

34. Guzmán BLB, Nava GT, Bevilacqua PD. Vigilância da qualidade da água para consumo humano: avaliando - grau de implementação das ações. Ciência Saúde Coletiva. 2014;19:4163-80. http://dx.doi.org/10.1590/1413812320141910.09452014

35. Gasselin P, Marrison K, Lapinte S, Valcke M. Avaliação de risco e epidemiologia ambiental: os novos desafios nas Américas. In: Galvao LA, Finkelman J, Henao S, editores. Determinantes ambientais e sociais da saúde. Washington, D.C.: Organização Pan-Americana da Saúde/Editora Fiocruz; 2011. p. 67-100.

36. Schütz G, Hacon S, Silva H, Moreno-Sánchez AR, Nagatani K. Principales marcos conceptuales aplicados para la evaluación de la salud ambiental mediante indicadores en América Latina y el Caribe. Rev Panam Salud Pública. 2008;24:276-85. http://dx.doi.org/10.1590/ S1020-49892008001000008 